\title{
CIRCULAR MOTION OF A PARTICLE UNDER FRICTION AND HYDRAULIC DISSIPATION
}

\author{
GIOVANNI MINGARI SCARPELLO AND DANIELE RITELLI
}

\begin{abstract}
A heavy particle travels along a rough circular path on a horizontal plane. Launched with a non-zero initial speed, due to the absence of other acting forces, but in presence of friction, it will slow down and eventually stop. The relevant transient is then solved in terms of the Jacobian elliptic functions of the time. Hydraulic air drag is the considered and the new resulting motion is ruled by the first order nonlinear ODE
\end{abstract}

$$
\dot{w}=-r w^{2}-C \sqrt{1+w^{4}}
$$

$(r, C)>0$, which is solved by means of the first kind Appell-Picard hypergeometric function of two variables $F_{1}\left(a, b, b^{\prime} ; c ; x, y\right)$.

\section{Introduction}

The cases in which the Ordinary Differential Equations (ODE) of the analytical Dynamics can be integrated in closed form, or solved by quadrature, are quite limited. Then the concept of "integrability", Liouville (1855), took place with his definition of integrable $n$ - Hamiltonian system in the unknown functions $x_{j}(t), j=1, \ldots, n$, based on the so called first integrals. These are some (known) functional relationships $\Phi_{i}\left(x_{1}, x_{2}, \ldots, x_{n}\right)$ taking a constant value ${ }^{1}$ when the Lagrangian coordinates $x_{j}$ satisfy the ODE motion.

A $n$-system is then considered (fully) integrable if one knows $n$ first integrals of it.

The Liouville definition of integrable systems covers: Keplerian motion; the harmonic oscillators, solved by trigonometric functions; rigid bodies (spinning tops) rotating about a fixed point- both the Euler-Poinsot and the Lagrange types- all described by elliptic functions. Another well known item is the pendulum, which is solved by special functions. These are: the elliptic functions for treating its large oscillations and complete revolutions $^{2}$; the Bessel functions for the variable length pendulum; finally, the Mathieu functions when the pendulum oscillates from a vibrating base.

Received February 11, 2003; revised February 20, 2004.

2000 Mathematics Subject Classification. Primary. 34A05; Secondary. 33E05, 33C65.

Key words and phrases. Analitycal Dynamics, Jacobi elliptic functions, Appell generalized hypergeometric function.

Research supported by MURST grant: Metodi matematici in economia.

${ }^{1}$ Of course $i$ is an integer such that $1<i \leq n$. Several questions, like the three-body problem, couldn't be completely solved for being known a number of first integrals $i<n$.

${ }^{2}$ Or those concerning the Watt flyball (centrifugal) governor: see [6]. 
At a higher level we can see geodetic motion over an ellipsoid, or the one-dimensional heat conduction through a radiating wire, or Kovalevskaya's case of the rotation of a rigid body about a fixed point, all needing hyperelliptic functions.

The Authors have been involved, for some time, in the field of integrable systems, with and without friction, and they have contributed some closed form integrations of ODE by means of higher transcendental functions $[6,7,8]$.

Here the (non-Hamiltonian) direct ${ }^{3}$ problem of interest has one degree of freedom namely the variable $(\vartheta)$ : it consists of a particle moving along a rough circular path. First it will be studied in vacuo (no air resistance); the air drag will then be added.

\section{In vacuo}

\subsection{The equations of motion}

A particle of mass $m$ immersed in a gravitational field $g$, is pulsed by a given initial speed. Then it turns in vacuo along a rough, circular path, on a horizontal plane, of center $O$ and radius $R$.

The system could be simulated by taking a small sphere, drilling a hole (from side to side) through it and introducing a thin wire into the hole, which is then bent to form a circle.

In order to ascertain the motion's time law, we will assume that the sense of the motion is counterclockwise, starting from $A$, with respect to a reference system whose co- mobile intrinsic frame $(\vec{t}, \vec{n}, \vec{b})$ is according to the Figure 1 . Projecting the equation $m \vec{a}=\vec{F}+\vec{\Phi}$ on the intrinsic axes, we obtain:

$$
\left\{\begin{array}{l}
m \ddot{s}=\Phi_{t} \\
m \frac{\dot{s}^{2}}{R}=\Phi_{n} \\
m g=\Phi_{b}
\end{array}\right.
$$

where $s$ is the curvilinear abscissa $s=\widehat{A P}(t)$. The equations of motion can also be written with respect to the angle $\vartheta$ :

$$
\left\{\begin{array}{l}
m R \ddot{\vartheta}=\Phi_{t} \\
m R \dot{\vartheta}^{2}=\Phi_{n} \\
m g=\Phi_{b}
\end{array}\right.
$$

$\Phi_{t}, \Phi_{n}$ and $\Phi_{b}$ being the components of the constraint reaction $\vec{\Phi}$ along the intrinsic frame $(\vec{t}, \vec{n}, \vec{b})$.

\footnotetext{
${ }^{3}$ Given the acting forces and the initial conditions (position and speed) of a system, the direct problem of Dynamics consists of detecting the motion, integrating the time equation and defining the trajectory. On the contrary, an inverse problem consists of determining the forces and the moments acting on a mobile body, from given kinematic elements of its motion.
} 


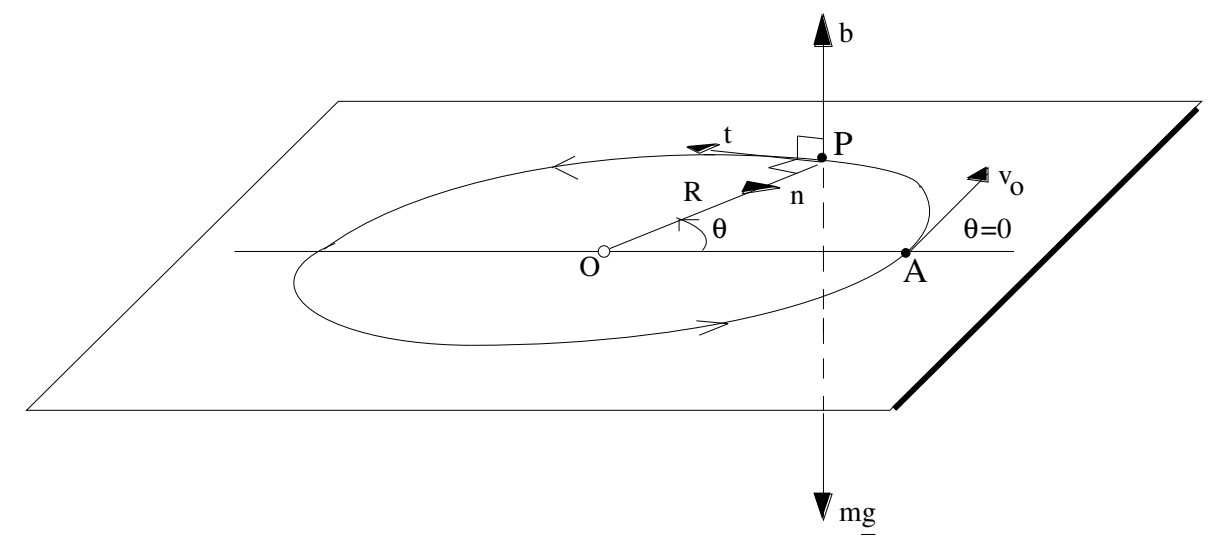

Figure 1. System's geometrical sketch.

\subsection{The main result}

After establishing the motion scalar equations (2.2), we can state the main result for the particle's motion in vacuo.

Theorem 2.1. Assume $\vartheta(0)=0$ and $\dot{\vartheta}(0)=\dot{\vartheta}_{0}=\frac{v_{0}}{R}$. Denote by $f>0$ the adimensional dynamic friction coefficient and

$$
C=\sqrt{\frac{g}{R}} f>0 .
$$

Finally, let ${ }^{4}$ :

$$
\mu_{0}=F\left(\arccos \frac{1-w_{0}^{2}}{1+w_{0}^{2}}, \frac{1}{\sqrt{2}}\right)
$$

where $w_{0}=\sqrt{\frac{R}{g}} \dot{\vartheta}_{0}$. Then the anomaly $\vartheta(t)$ of (2.2)-which we will prove physically defined for $0 \leq t \leq \frac{\mu_{0}}{2 C}-$ grows as the logarithm of the Jacobi cosinus amplitude (cn) of the time $e^{5}$ :

$$
\vartheta(t)=\frac{\sqrt{R}}{f} \ln \left[\frac{\operatorname{cn}\left(\frac{\mu_{0}}{2}-C t\right)}{\operatorname{cn} \frac{\mu_{0}}{2}}\right] .
$$

The proof of Theorem 2.1 is quite elaborate, so we shall split it into several steps. Due to the circular trajectory:

$$
\dot{\vartheta}=\frac{v}{R}, \quad \ddot{\vartheta}=\frac{\dot{v}}{R}
$$

${ }^{4} F(\varphi, k)$ denotes the canonical (incomplete) elliptic integral of the first kind, of amplitude $\varphi$ and modulus $k$.

${ }^{5}$ Its modulus $1 / \sqrt{2}$ is for simplicity omitted. 
Then (2.2) becomes:

$$
\left\{\begin{array}{l}
m \dot{v}=\Phi_{t} \\
m \frac{v^{2}}{R}=\Phi_{n} \\
m g=\Phi_{b}
\end{array}\right.
$$

The law of motion with dynamic friction gives:

$$
\Phi_{t}=\mp f \sqrt{\Phi_{n}^{2}+\Phi_{b}^{2}}
$$

then we obtain a nonlinear first order ODE in $v$ which is pure (the reactive components do not appear) and independent of the mass:

$$
\frac{\dot{v}}{\sqrt{g R}}=-\frac{g f}{\sqrt{g R}} \sqrt{1+\frac{v^{4}}{(g R)^{2}}} .
$$

The minus sign has been selected because - due to the absence of propelling forces - the motion takes place with a speed, monotonically decreasing with time. Setting $\frac{v}{\sqrt{g R}}=w$ (adimensional speed), we obtain the equation:

$$
\dot{w}=-C \sqrt{1+w^{4}},
$$

which shall be solved with the initial condition:

$$
w(0)=\frac{v_{0}}{\sqrt{g R}}=\sqrt{\frac{R}{g}} \dot{\vartheta}_{0}=w_{0} .
$$

Equation (2.8) is separable:

$$
-C t=\int_{w_{0}}^{w} \frac{d \xi}{\sqrt{1+\xi^{4}}} .
$$

The integral at the right hand side of $(2.10)$ is elliptic ${ }^{6}$ in order to invert it and to solve for $w$, we recall the following Lemma 2.1 (see also [4] page $262 \mathrm{n}^{\circ} 10$ ).

Lemma 2.1. The following integral formulae hold:

$$
\begin{aligned}
\int_{0}^{u} \frac{d \xi}{\sqrt{1+\xi^{4}}} & =\frac{1}{2} F\left(\arccos \frac{1-u^{2}}{1+u^{2}}, \frac{1}{\sqrt{2}}\right) \\
\int_{0}^{u} \sqrt{1+\xi^{4}} d \xi & =\frac{1}{3} u \sqrt{1+u^{4}}+\frac{1}{3} F\left(\arccos \frac{1-u^{2}}{1+u^{2}}, \frac{1}{\sqrt{2}}\right) .
\end{aligned}
$$

Proof. Setting:

$$
\frac{1-\xi^{2}}{1+\xi^{2}}=\cos \psi
$$

${ }^{6}$ We have $\sqrt{1+w^{4}}$ instead of $w^{2}$ as a consequence of the nonzero gravity! See, for a detailed discussion, $\S 2.2$. 
we obtain:

$$
\xi=\sqrt{\frac{1-\cos \psi}{1+\cos \psi}}=\tan \frac{\psi}{2}
$$

and then:

$$
\int_{0}^{u} \frac{d \xi}{\sqrt{1+\xi^{4}}}=\frac{1}{2} \int_{0}^{\arccos \frac{1-u^{2}}{1+u^{2}}} \frac{d \psi}{\sqrt{\cos ^{4} \frac{\psi}{2}+\sin ^{4} \frac{\psi}{2}}} .
$$

Now, recalling that:

$$
\sin ^{4} \frac{\psi}{2}+\cos ^{4} \frac{\psi}{2}=\left(\sin ^{2} \frac{\psi}{2}+\cos ^{2} \frac{\psi}{2}\right)^{2}-2 \sin ^{2} \frac{\psi}{2} \cos ^{2} \frac{\psi}{2},
$$

we can write:

$$
\cos ^{4} \frac{\psi}{2}+\sin ^{4} \frac{\psi}{2}=1-\frac{1}{2} \sin ^{2} \psi
$$

This proves (2.11). The proof of (2.12) now follows; integrating by parts we find:

$$
\int_{0}^{u} \sqrt{1+\xi^{4}} d \xi=u \sqrt{1+u^{4}}-2 \int_{0}^{u} \frac{\xi^{4}}{\sqrt{1+\xi^{4}}} d \xi
$$

but

and so:

$$
\frac{\xi^{4}}{\sqrt{1+\xi^{4}}}=\sqrt{1+\xi^{4}}-\frac{1}{\sqrt{1+\xi^{4}}}
$$

$$
\int_{0}^{u} \sqrt{1+\xi^{4}} d \xi=u \sqrt{1+u^{4}}-2 \int_{0}^{u} \sqrt{1+\xi^{4}}+2 \int_{0}^{u} \frac{d \xi}{\sqrt{1+\xi^{4}}}
$$

which gives (2.12).

Using Lemma 2.1 we can formulate a second Lemma 2.2.

Lemma 2.2. The solution of (2.8), with initial datum given by (2.9), is:

$$
w(t)=\sqrt{\frac{1-\mathrm{cn}\left(\mu_{0}-2 C t, \frac{1}{\sqrt{2}}\right)}{1+\mathrm{cn}\left(\mu_{0}-2 C t, \frac{1}{\sqrt{2}}\right)}}, 0 \leq t \leq \frac{\mu_{0}}{2 C} .
$$

Proof. We can write (2.10) as:

$$
-C t+\int_{0}^{w_{0}} \frac{d \xi}{\sqrt{1+\xi^{4}}}=\int_{0}^{w} \frac{d \xi}{\sqrt{1+\xi^{4}}}
$$

therefore we have, using Lemma 2.1:

$$
-C t+\frac{1}{2} \mu_{0}=\frac{1}{2} F\left(\arccos \frac{1-w^{2}}{1+w^{2}}, \frac{1}{\sqrt{2}}\right) .
$$


The left hand side of (2.13) is not negative, since $0 \leq t \leq \frac{\mu_{0}}{2 C}$. Introducing the quantity:

$$
\psi=\arccos \frac{1-w^{2}}{1+w^{2}},
$$

we have:

$$
\psi=\operatorname{am}\left(\mu_{0}-2 C t, \frac{1}{\sqrt{2}}\right)
$$

Noticing that:

$$
\cos \psi=\frac{1-w^{2}}{1+w^{2}}=\mathrm{cn}\left(\mu_{0}-2 C t, \frac{1}{\sqrt{2}}\right),
$$

we finally can write the solution of (2.8):

$$
w(t)=\sqrt{\frac{1-\mathrm{cn}\left(\mu_{0}-2 C t, \frac{1}{\sqrt{2}}\right)}{1+\mathrm{cn}\left(\mu_{0}-2 C t, \frac{1}{\sqrt{2}}\right)}} .
$$

It is now easy to compute the dimensional speed from (2.7):

$$
v(t)=\sqrt{g R} w(t)
$$

The motion will reach the equilibrium after a time $t_{S}$ such that:

$$
1-\operatorname{cn}\left(\mu_{0}-2 C t_{S}, \frac{1}{\sqrt{2}}\right)=0 .
$$

Recalling that the cosinus amplitude has value 1 when its argument is zero:

$$
t_{S}=\frac{\mu_{0}}{2 C}=\frac{F\left(\arccos \frac{1-\frac{v_{0}^{2}}{R g}}{1+\frac{v_{0}^{2}}{R g}}, \frac{1}{\sqrt{2}}\right)}{\frac{2 f \sqrt{g}}{R}} .
$$

Therefore $t_{S}$ does not depend upon the mass of the particle but on the radius; on the contrary, when the dynamic friction $f \rightarrow 0^{+}$, then $t_{S} \rightarrow \infty$. Moreover $t_{S} \rightarrow \infty$ as $R \rightarrow \infty$ since:

$$
t_{S} \sim \frac{\sqrt{R} v_{0}}{f g}+\mathrm{O}\left(R^{-1 / 2}\right) .
$$

Therefore, increasing the radius, the slowing time increases too, as suggested by physical intuition.

We must restrict our time span from zero to $\frac{\mu_{0}}{2 C}$, while the ODE (2.10) is mathematically defined also for greater values of $t$. The motion we are dealing with, is pulsed by the initial conditions only (position and speed). Due to speed, the particle has a kinetic 
energy which will decrease during its motion if not affected by any external force, but contrasted by passive drag. It is then physically consequent that the solution of (2.8) has to be considered, not for any $t$, but only until $t_{S}=\frac{\mu_{0}}{2 C}$, as previously said. Beyond this value, negative meaningless solutions would appear; moreover we have, due to the Wintner Theorem (see [5] Theorem 5.1 pages 29-30), a blow up phenomenon: a positive value exists $t_{\omega}>t_{S}$ such that:

$$
\lim _{t \rightarrow t_{\omega}} w(t)=-\infty
$$

It is now possible to complete the proof of Theorem 2.1 integrating the speed expressed by $(2.14)$.

Proof.(Of Theorem 2.1). Dropping the modulus specification $\frac{1}{\sqrt{2}}$, we have:

$$
\dot{\vartheta}(t)=\sqrt{\frac{g}{R}} \sqrt{\frac{1-\operatorname{cn}\left(\mu_{0}-2 C t\right)}{1+\operatorname{cn}\left(\mu_{0}-2 C t\right)}}, \quad 0 \leq t \leq \frac{\mu_{0}}{2 C} .
$$

We recall the following identity, [2] page 25, $\mathrm{n}^{\circ} 129.04$ :

$$
\frac{1-\operatorname{cn} 2 u}{1+\operatorname{cn} 2 u}=\operatorname{tn}^{2} u \operatorname{dn}^{2} u
$$

so we can write:

$$
\dot{\vartheta}=\sqrt{\frac{g}{R}} \operatorname{tn}\left(\frac{\mu_{0}}{2}-C t\right) \operatorname{dn}\left(\frac{\mu_{0}}{2}-C t\right) .
$$

Now by the indefinite integration formula:

$$
\int \operatorname{tn} u \operatorname{dn} u d u=-\ln \operatorname{cn} u+K
$$

we find, for $0 \leq t \leq \frac{\mu_{0}}{2 C}$ :

$$
\int \operatorname{tn}\left(\frac{\mu_{0}}{2}-C t\right) \operatorname{dn}\left(\frac{\mu_{0}}{2}-C t\right) d t=\frac{\ln \operatorname{cn}\left(\frac{\mu_{0}}{2}-C t\right)}{C}+K .
$$

Finally, to integrate $(2.15)$ we must take into account the initial datum $\vartheta(0)=0$, and recalling (2.3), we obtain:

$$
\vartheta(t)=\frac{\sqrt{R}}{f} \ln \left[\frac{\operatorname{cn}\left(\frac{\mu_{0}}{2}-C t\right)}{\operatorname{cn}\left(\frac{\mu_{0}}{2}\right)}\right],
$$

where $0 \leq t \leq \frac{\mu_{0}}{2 C}$.

Remark. From the mechanical point of view, the effect of the gravitational field could be deemed as "minor", the relevant force on $m$ being perpendicular to the motion plane, and thus unable to affect its motion itself. Such a conclusion would be wrong. 
Gravity is the main cause ${ }^{7}$ for the complexity of motion. To check this, let us remove gravity, putting $g=0$ in (2.6). We obtain:

$$
\left\{\begin{array}{l}
\frac{d v}{v^{2}}=-\frac{f}{R} d t \\
v(0)=v_{0}
\end{array}\right.
$$

namely:

$$
\frac{1}{v}-\frac{1}{v_{0}}=\frac{f}{R} t
$$

Replacing $v=R \dot{\vartheta}$, and integrating again with $\vartheta(0)=\vartheta_{0}$, we get:

$$
\vartheta(t)=\vartheta_{0}+\frac{1}{f} \ln \left(1+f \dot{\vartheta}_{0} t\right) .
$$

Then the nonlinear motion would collapse into an elementary one, if -cœeteris paribus and in the presence of dry friction too - the particle was no longer subjected to gravity.

\subsection{An example}

The solution of equation (2.8) is not affected by bifurcations and there is no critical value for the parameters. Then we set all constants to the value 1 . The solution blows up in any case, see for instance [5], but the model has a physical meaning only for $t \leq t_{S}$.

In the following Figure 2 we superimposed our exact solution $w(t)$ of $(2.8)$, considered with $g=R=f=v_{0}=1$, to the output of standard numerical methods provided by Mathematica $(\mathbb{R}$. More precisely, we used the package VisualDSolve.m developed by [9]. The outcome is satisfactory. The computed $t_{S}$ is 0.927566 . The elliptic function $\vartheta(t)$ (2.5), is shown in Figure 3. The anomaly $\vartheta$ increases with time, but at a progressively slower rate, due to the fact that the slope of $w(t)$ tends to zero.

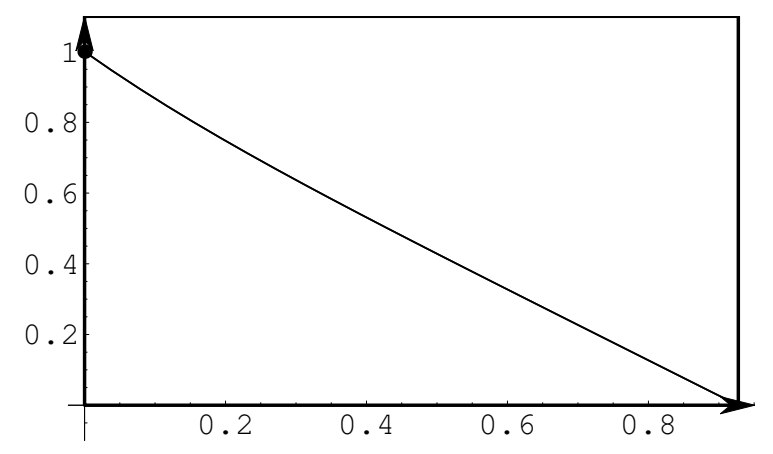

Figure 2. Adimensional speed of the particle versus time.

\footnotetext{
${ }^{7}$ By the third equation in (2.2) we see that $g$ affects the binormal reactive component $\Phi_{b}$, and then, through it, $\Phi_{t}$, and so, finally $\dot{v}$.
} 


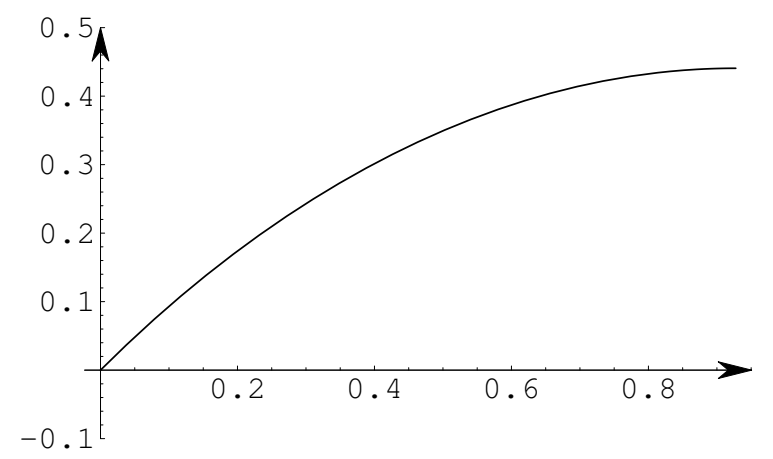

Figure 3. Particle anomaly (2.5) versus time.

\section{Hydraulically dissipative medium}

\subsection{The equations of motion}

Adding air hydraulic resistance introduces a supplementary force, varying as $\dot{s}^{2}$ and having the same instantaneous direction (but reverse sense) of the $\vec{t}$ vector; then only the first equation in (2.1) will be changed:

$$
\left\{\begin{array}{l}
m \ddot{s}=\Phi_{t}-k \dot{s}^{2} \\
m \frac{\dot{s}^{2}}{R}=\Phi_{n} \\
m g=\Phi_{b}
\end{array}\right.
$$

where $k$ is called the hydraulic resistance coefficient. The motion can also be described in terms of the angle $\vartheta$ :

$$
\left\{\begin{array}{l}
m R \ddot{\vartheta}=\Phi_{t}-k R^{2} \dot{\vartheta}^{2} \\
m R \dot{\vartheta}^{2}=\Phi_{n} \\
m g=\Phi_{b}
\end{array}\right.
$$

or, as for (2.6):

$$
\left\{\begin{array}{l}
m \dot{v}+k v^{2}=\Phi_{t} \\
m \frac{v^{2}}{R}=\Phi_{n} \\
m g=\Phi_{b}
\end{array}\right.
$$

In this way we obtain the ODE of motion for the heavy rotating point under both dry friction (constraint) and hydraulic drag (medium):

$$
\frac{\dot{v}}{\sqrt{R g}}+\frac{k}{m} \sqrt{R g}(\dot{v} / \sqrt{R g})^{2}=-\frac{g f}{\sqrt{g R}} \sqrt{1+(v / \sqrt{R g})^{4}} .
$$


Equation (3.1) differs from (2.7) only in the term:

$$
\frac{k}{m} \sqrt{R g}(\dot{v} / \sqrt{R g})^{2}=r(\dot{v} / \sqrt{R g})^{2}
$$

where we set:

$$
r=\frac{k}{m} \sqrt{R g}>0
$$

\subsection{Explicit integration}

The explicit integration will be conveniently carried out considering the variant of $(3.1)$ :

$$
\dot{w}=-r w^{2}-C \sqrt{1+w^{4}},
$$

in the adimensional variable $w=\frac{v}{\sqrt{R g}}(C$ as in Theorem 2.1). The differential equation (3.2) is separable. Before integrating it, we establish Lemma 3.1.

Lemma 3.1. If $p$ and $q$ are two real numbers such that, if $p q>0$, the real root of the equation $p x^{4}-q=0$ lies outside the interval $[\alpha, \beta]$, then the following relation holds:

$\int_{\alpha}^{\beta} \frac{\sqrt{1+x^{4}}}{p x^{4}-q} d x=\frac{1}{q}\left\{\alpha F_{1}\left(\frac{1}{4}, 1,-\frac{1}{2} ; \frac{5}{4} ;-\alpha^{4}, \frac{p}{q} \alpha^{4}\right)+-\beta F_{1}\left(\frac{1}{4}, 1,-\frac{1}{2} ; \frac{5}{4} ;-\beta^{4}, \frac{p}{q} \beta^{4}\right)\right\}$.

Here $F_{1}\left(a, b, b^{\prime} ; c ; x, y\right)$ is the first kind ${ }^{8}$ Appell [1] generalised hypergeometric function; this requires that all the following inequalities are fulfilled:

$$
|\alpha|<1, \frac{p}{q} \alpha^{4}<1,|\beta|<1, \frac{p}{q} \beta^{4}<1
$$

Proof. We recall the 4-parameter, 2-variable Appell hypergeometric function $F_{1}(a, b$, $\left.b^{\prime} ; c ; x, y\right)$ given as a double power series:

$$
F_{1}\left(a, b, b^{\prime} ; c ; x, y\right)=\sum_{m=0}^{\infty} \sum_{n=0}^{\infty} \frac{(a)_{m+n}(b)_{m}\left(b^{\prime}\right)_{n}}{(c)_{m+n}} \frac{x^{m}}{m !} \frac{y^{n}}{n !},
$$

where $(a)_{m+n},(b)_{m},\left(b^{\prime}\right)_{n},(c)_{m+n}$ are Pochhammer symbols. The series converges for $|x|<1,|y|<1$. We refer to $x$ and $y$ as the arguments of $F_{1}$. The starting point for proving Lemma 3.1 is the possibility of making use of the integral representation of the Appell hypergeometric function (see [3] page 27, formula (1.4.2.6)):

$$
F_{1}\left(a, b, b^{\prime} ; c ; x, y\right)=\frac{\Gamma(c)}{\Gamma(a) \Gamma(c-a)} \mathcal{I}
$$

${ }^{8}$ From the hypergeometric functions ${ }_{p} F_{q}(\ldots ; x)$ (with several parameters but) of single argument $x$, Appell in 1880 arrived at a generalisation involving the double series. He treated this matter on a systematic basis and defined four functions bearing his name. All of them have 2 arguments; furthermore $F_{1}$ and $F_{4}$ have four parameters, whilst $F_{2}$ and $F_{3}$ have five parameters. See [10]. 
where:

$$
\mathcal{I}=\int_{0}^{1} u^{a-1}(1-u)^{c-a-1}(1-u x)^{-b}(1-u y)^{-b^{\prime}} d u ; a>0, c-a>0 .
$$

First let us perform the change of variable $x=\sqrt[4]{u}$ :

$$
\int_{\alpha}^{\beta} \frac{\sqrt{1+x^{4}}}{p x^{4}-q} d x=-\frac{1}{4 q} \int_{\alpha^{4}}^{\beta^{4}} u^{-\frac{3}{4}}[1-(-u)]^{\frac{1}{2}}\left(1-\frac{p}{q} u\right)^{-1} d u .
$$

So we see that the sequence of the parameters is:

$$
\left\{\begin{array} { l } 
{ a - 1 = - \frac { 3 } { 4 } , } \\
{ c - a - 1 = 0 , } \\
{ - b _ { 1 } = - 1 } \\
{ - b _ { 2 } = \frac { 1 } { 2 } . }
\end{array} \leadsto \left\{\begin{array}{l}
a=\frac{1}{4}, \\
c=\frac{5}{4}, \\
b_{1}=1, \\
b_{2}=-\frac{1}{2} .
\end{array}\right.\right.
$$

Now we introduce, for simplicity $h(u)=u^{-\frac{3}{4}}[1-(-u)]^{\frac{1}{2}}\left(1-\frac{p}{q} u\right)^{-1}$, then:

$$
\int_{\alpha}^{\beta} \frac{\sqrt{1+x^{4}}}{p x^{4}-q} d x=\frac{1}{4 q}\left\{\int_{0}^{\alpha^{4}} h(u) d u-\int_{0}^{\beta^{4}} h(u) d u\right\}=\frac{1}{4 q}\{M-N\} .
$$

To apply the integral representation, we need to normalise the integrals through some suitable change of variable.

Setting $v=\frac{1}{\alpha^{4}} u$ and taking into account that $\frac{\Gamma\left(\frac{1}{4}\right) \Gamma\left(\frac{5}{4}-\frac{1}{4}\right)}{\Gamma\left(\frac{5}{4}\right)}=4$, we have:

$$
\begin{aligned}
M & =\alpha \int_{0}^{1} v^{-\frac{3}{4}}\left(1+\alpha^{4} v\right)^{\frac{1}{2}}\left(1-\frac{p}{q} \alpha^{4} v\right)^{-1} d v \\
& =4 \alpha F_{1}\left(\frac{1}{4}, 1,-\frac{1}{2} ; \frac{5}{4} ;-\alpha^{4}, \frac{p}{q} \alpha^{4}\right) .
\end{aligned}
$$

In the same way, if $v=\frac{1}{\beta^{4}} u$ we have:

$$
\begin{aligned}
N & =\beta \int_{0}^{1} v^{-\frac{3}{4}}\left(1+\beta^{4} v\right)^{\frac{1}{2}}\left(1-\frac{p}{q} \beta^{4} v\right)^{-1} d v \\
& =4 \beta F_{1}\left(\frac{1}{4}, 1,-\frac{1}{2} ; \frac{5}{4} ;-\beta^{4}, \frac{p}{q} \beta^{4}\right) .
\end{aligned}
$$

Lemma 3.1 is then proved.

Now we will use Lemma 3.1 for (3.2), performing the integration and providing a relationship $t=H(w)$, because the inverse $w=H^{-1}(t)$ cannot be expressed via known functions. We will assume the initial value $w_{0}=w(0)=\frac{v_{0}}{\sqrt{R g}}$. 
Theorem 3.1. (i) If $r>C$ and:

$$
w_{0}<\min \left\{1, \sqrt[4]{\frac{C^{2}}{r^{2}-C^{2}}}\right\},
$$

the time equation, solution of (3.2), is given by:

$$
t=H_{1}(w)+H_{2}(w),
$$

where:

$$
\begin{gathered}
H_{1}(w)=\frac{r}{r^{2}-C^{2}} \frac{1}{2 A}\left\{\frac{1}{2} \ln \frac{\left(w_{0}+A\right)(w-A)}{\left(w_{0}-A\right)(w+A)}+\arctan \frac{w}{A}-\arctan \frac{w_{0}}{A}\right\} \\
H_{2}(w)=\frac{1}{C^{2}}\left\{w_{0} F_{1}\left(\frac{1}{4}, 1,-\frac{1}{2} ; \frac{5}{4} ;-w_{0}^{4}, \frac{r^{2}-C^{2}}{C^{2}} w_{0}^{4}\right)\right. \\
\left.\quad-w F_{1}\left(\frac{1}{4}, 1,-\frac{1}{2} ; \frac{5}{4} ;-w^{4}, \frac{r^{2}-C^{2}}{C^{2}} w^{4}\right)\right\}
\end{gathered}
$$

where, for brevity, we set:

$$
A=\sqrt[4]{\frac{C^{2}}{r^{2}-C^{2}}}
$$

(ii) If $r<C$ and:

$$
w_{0}<1
$$

in $t=H_{1}(w)+H_{2}(w)$ we have to take:

$$
\begin{gathered}
H_{1}(w)=\frac{r}{r^{2}-C^{2}} \frac{1}{2 \sqrt{2} A}\left\{\frac{1}{2} \ln \frac{\left(w_{0}^{2}+\sqrt{2} A w_{0}+A^{2}\right)\left(w^{2}-\sqrt{2} A w_{0}+A^{2}\right)}{\left(w_{0}^{2}-\sqrt{2} A w_{0}+A^{2}\right)\left(w^{2}+\sqrt{2} A w_{0}+A^{2}\right)}\right. \\
\left.+\arctan \frac{2 w+\sqrt{2} A}{\sqrt{2} A}-\arctan \frac{2 w_{0}+\sqrt{2} A}{\sqrt{2} A}\right\} \\
H_{2}(w)=\frac{1}{C^{2}}\left\{w_{0} F_{1}\left(\frac{1}{4}, 1,-\frac{1}{2} ; \frac{5}{4} ;-w_{0}^{4}, \frac{r^{2}-C^{2}}{C^{2}} w_{0}^{4}\right)\right. \\
\left.-w F_{1}\left(\frac{1}{4}, 1,-\frac{1}{2} ; \frac{5}{4} ;-w^{4}, \frac{r^{2}-C^{2}}{C^{2}} w^{4}\right)\right\}
\end{gathered}
$$

where we set:

$$
A=\sqrt[4]{\frac{C^{2}}{C^{2}-r^{2}}} .
$$

(iii) Finally, if $r=C$ the solution of (3.2) is:

$$
\begin{aligned}
t=-\frac{1}{3 C} & \left\{w^{3}-w \sqrt{1+w^{4}}-F\left(\arccos \frac{1-w^{2}}{1+w^{2}}, \frac{1}{\sqrt{2}}\right)\right. \\
& \left.-w_{0}^{3}+w_{0} \sqrt{1+w_{0}^{4}}+F\left(\arccos \frac{1-w_{0}^{2}}{1+w_{0}^{2}}, \frac{1}{\sqrt{2}}\right)\right\} .
\end{aligned}
$$


Proof. We have already noticed that (3.2) is separable. Its solution then will be:

$$
\begin{aligned}
-t & =\int_{w_{0}}^{w} \frac{d \xi}{r \xi^{2}+C \sqrt{1+\xi^{4}}} \\
& =r \int_{w_{0}}^{w} \frac{\xi^{2}}{\left(r^{2}-C^{2}\right) \xi^{4}-C^{2}} d \xi-C \int_{w_{0}}^{w} \frac{\sqrt{1+\xi^{4}}}{\left(r^{2}-C^{2}\right) \xi^{4}-C^{2}} d \xi \\
& =r \mathcal{I}_{1}-C \mathcal{I}_{2} .
\end{aligned}
$$

Let us assume now that $r>C$ holds. In this case we must assume that $\left(r^{2}-C^{2}\right) w_{0}^{4}-$ $C^{2} \neq 0$. Then: for any $w$ such that $\left(r^{2}-C^{2}\right) w^{4}-C^{2} \neq 0$, we can perform the integration. The integral $\mathcal{I}_{1}$ can be computed after reducing it in partial fractions:

$$
\frac{\xi^{2}}{\xi^{4}-A^{4}}=\frac{1}{4 A}\left(\frac{1}{\xi-A}-\frac{1}{\xi+A}\right)+\frac{1}{2\left(\xi^{2}+A^{2}\right)},
$$

where $A^{4}=\frac{C^{2}}{r^{2}-C^{2}}>0$. The second integral is computed by exploiting Lemma 3.1 where $p=r^{2}-C^{2}$ and $q=C^{2}$.

If $r<C$, the proof is analogous, the only difference being that we have no restriction on $w_{0}$ and $w$. Taking $A=\frac{c^{2}}{c^{2}-r^{2}}$ the evaluation of $\mathcal{I}_{1}$ follows from the identity:

$$
\frac{\xi^{2}}{\xi^{4}+A^{4}}=\frac{1}{2 \sqrt{2} A}\left\{\frac{\xi}{\xi^{2}-\sqrt{2} A+A^{2}}-\frac{\xi}{\xi^{2}+\sqrt{2} A+A^{2}}\right\},
$$

while $\mathcal{I}_{2}$ is exactly the same as before.

The degenerate ${ }^{9}$ case $r=C$ follows from the second part of Lemma 2.1, in fact we have:

$$
t=-\frac{1}{C} \int_{w_{0}}^{w} \frac{d \xi}{\xi^{2}+\sqrt{1+\xi^{4}}}=-\frac{1}{C} \int_{w_{0}}^{w}\left(\sqrt{1+\xi^{4}}-\xi^{2}\right) d \xi
$$

The second integral is elementary while the first one was already computed (formula (2.12)). The statement is then fully proved.

\section{Remarks.}

1. In any case, each solution $t=t(w)$ will be meaningful for speeds decreasing from $w=w_{0}$ (startup) to $w=0$ (rest).

2. Formulae like $t=t(w)$ allow a (relatively) simple answer to the question: when will the mobile attain a prescribed speed while slowing down? The reverse question of finding the speed at an assigned time, is, of course, a numerical matter.

3. The "modelling" of the integral given by Lemma 3.1 through the Appell I kind hypergeometric function $F_{1}$, is correct if, and only if, the modulus of the (variable) arguments of $F_{1}$ is $<1$. This is the meaning of the hypotheses (3.3) and (3.4). When

\footnotetext{
${ }^{9}$ Degeneracy means that the generalised hypergeometric function is replaced by its special case given by the first kind elliptic integral.
} 
these conditions are not met, one has to consider the motion expressed by the definite integral:

$$
\int_{w_{0}}^{w} \frac{\sqrt{1+\xi^{4}}}{\left(r^{2}-C^{2}\right) \xi^{4}-C^{2}} d \xi
$$

holds true, but it is not representable any more by means of the Appell function $F_{1}$.

4. It is soon seen that the three examined occurrences

$$
r \gtreqless C
$$

can be summarized by the inequality:

$$
\frac{k}{m} R \sqrt{R} \gtreqless f
$$

measuring the dissipation unbalance (or the balance) between the hydraulic resistance $k$ and the dry friction $f$, the particle $(m)$ and the ring $(R)$ being fixed. Then the above cases can be read as:

1. prevailingly hydraulic dissipation,

2. prevailingly frictional drag,

3. balanced dissipation.

\subsection{Examples}

We present, here, three applications of Theorem 3.1, showing the superimposition of our exact, closed form solutions with the relevant numerical outputs, independently obtained via Mathematica $\mathbb{R}$ [9]. As usual, we try to normalise the constant as much as possible: in each case the speed decreases, due to dissipation. To begin with $(i)$, let us show the solutions $(w, t)$ for $r>C$. We select $r=2, C=1, w_{0}=0.7$. For the case (ii), $r<C$ we choose $r=1, C=2, w_{0}=0.7$. Finally, in the case $(i i i), r=C$ we take: $r=1, C=1, w_{0}=0.7$. When double dissipation occurs, the slowing time can be evaluated only by means of numerical techniques. The blow up phenomenon still arises, due to the well known classical results in [5].

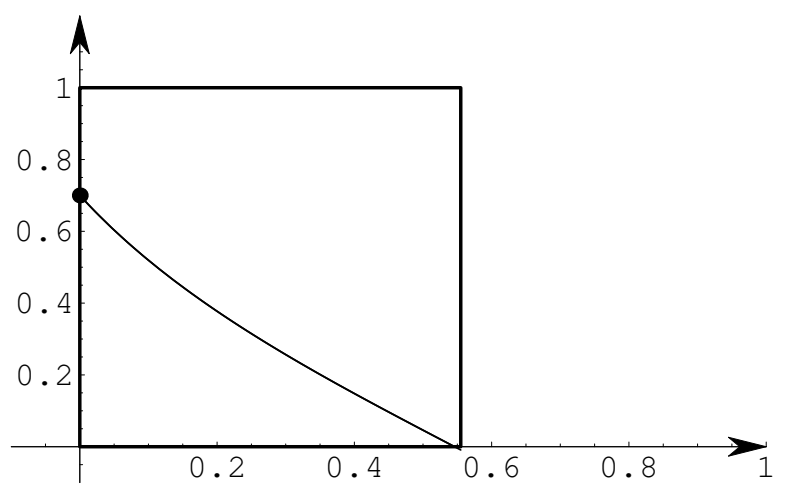

Figure 4. Speed decreases when the hydraulic dissipation prevails. 


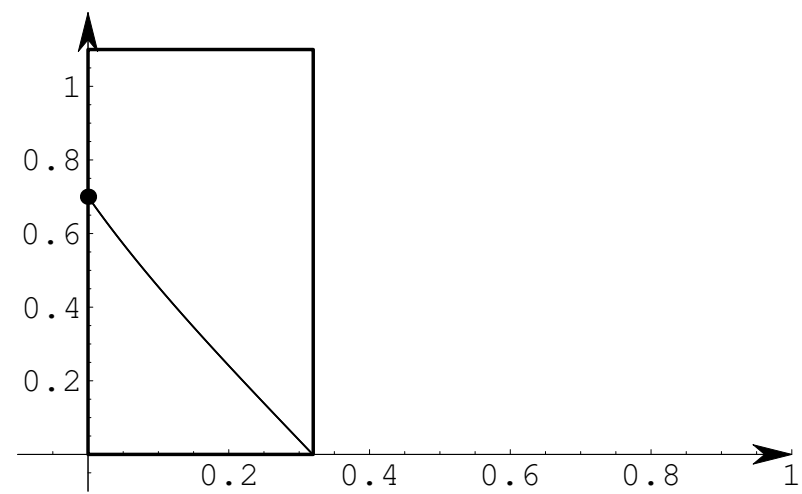

Figure 5. Speed decreases when the frictional drag prevails.

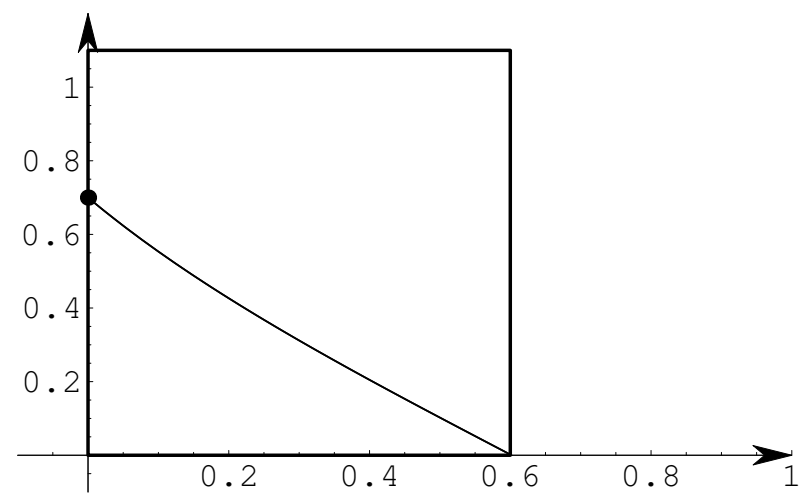

Figure 6. Speed decreases in presence of balanced dissipation.

\section{Conclusions}

In this problem we have set up a system consisting of:

1. a heavy punctual particle,

2. moving along the geometrically simplest, bounded trajectory,

3. in absence of active, propelling forces,

4. in vacuo,

5. under dry dynamic friction (due to the constraint),

which acquires, due to its non-hamiltonian nature (the friction), a first mathematical complexity ruled by elliptic functions. So we have arrived at a closed formula, see (2.5), 
of the anomaly $\vartheta$ as a logarithm of the cosinus amplitude of time; the time $t_{S}$ when the motion will be extinguished, has been formulated by means of the Legendre I kind elliptic integral.

Coeteris paribus, the model's complexity grows far more, see the nonlinear ODE (3.2), adding a further dissipation; consequently for its solution, the (much less frequently met) Appell function $F_{1}\left(a, b, b^{\prime} ; c ; x, y\right)$ enters the scene. A Theorem 3.1 has been established, with three different time equations $t=t(w)$ according to which quantity (either $k$ or $m$ ) is prevailing in the inequality:

$$
\frac{k}{m} R \sqrt{R} \gtreqless f
$$

between the hydraulic resistance $k$ and the dynamic friction $f$, for fixed particle $(m)$ and the radius $(R)$.

\section{Acknowledgements}

The authors are indebted to Aldo Scimone who drew up the first figure of this paper: they hereby take this opportunity to warmly thank him. They are also deeply grateful to Ruth Hodkinsons, Enrico Bernardi and Giulia Spaletta, who helped us in the final, formal revision of the text.

\section{References}

[1] P. Appell, Sur les séries hypergéométriques de deux variables, C. R. Acad. Sci. Paris Ser. I 90 (1880), 296-298.

[2] P. F. Byrd and M. D. Friedman, Handbook of Elliptic Integrals for Engineers and Scientists, Springer Verlag, Berlin, 1971.

[3] H. Exton, Multiple Hypergeometric Functions and applications. Ellis Horwood Limited, Chichester, 1976.

[4] I. S. Gradshteyn and I. M. Ryzhik, Table of Integrals, Series and Products, Springer-Verlag, New York, 1980.

[5] P. Hartman, Ordinary Differential Equations, John Wiley \& Sons, New York, 1964.

[6] G. Mingari Scarpello and D. Ritelli, Closed form integration of the rotating plane pendulum nonlinear equation, Tamkang J. Math. 34(2003), 327-350.

[7] G. Mingari Scarpello and D. Ritelli D, A nonlinear oscillation induced by two fixed gravitating centres, Int. Math. J. 4 (2003), 337-350.

[8] G. Mingari Scarpello and D. Ritelli, A nonlinear oscillator modeling the transverse vibration of a rod, Rend. Semin. Mat. Torino 61 (2003), 55-69.

[9] D. Schwalbe and S. Wagon, VisualDSolve, Springer, Berlin 1997.

[10] H. M. Srivastava and P. W. Karlsson, Multiple Gaussian Hypergeometric Series, Ellis Horwood Limited, Chichester, 1985.

Libero Università di Bolzano, Facoltà di Economia, via Sernesi 1, 39100 Bolzano, Italy.

E-mail: gmingariscarpello@unibz.it

Dipartimento di matematica per le scienze economiche e sociali, viale Filopanti, 540126 Bologna

Italy.

E-mail: dritelli@economia.unibo.it 